\title{
Environmental protection and social development: optimization perspective from two polarities
}

\author{
Qianyue $\mathrm{He}^{1}$, Zihao $\mathrm{Lei}^{2}$, Ruoding $\mathrm{An}^{1}$, Yiyun $\mathrm{Yu}^{1}$, Xin Huang ${ }^{2}$, Guangrui $\mathrm{Wen}^{2,}$, and Xuefeng Chen ${ }^{2}$ \\ ${ }^{1}$ School of Automation Science and Engineering, Xi'an Jiaotong University, Xi'an 710049, China \\ ${ }^{2}$ School of Mechanical Engineering, Xi'an Jiaotong University, Xi'an 710049, China
}

\begin{abstract}
The development of human society is currently in a dilemma, where the further development of society needs to be considered, simultaneously, the environmental problems caused by development are becoming increasingly prominent. Therefore, how to weigh the relationship between environmental protection and social development is still a hot research topic. In consideration of such a bipolar conflict problem, a novel optimization model was proposed to study the relationship between environmental protection and social development. First, this problem is modeled as a single objective optimization problem. Secondly, a series of representative characteristics are selected from social development and environmental protection. Then, these features are preprocessed, decomposed and analyzed. Finally, the objective function is obtained through regression analysis. In order to verify the effectiveness of the proposed approach, the realistic case of China from 2000 to 2018 are employed and analyzed. The analysis result confirms that proposed model is able to achieve state-of-the-art performances.
\end{abstract}

\section{Introduction}

As the process of industrialization continues to intensify, the pressure brought about by development is approaching the capacity of environment. As a result, many environmental problems have emerged, including climate warming, air pollution, water pollution and so on. At the same time, humans are also suffering from the cost of environmental degradation [1]. Among the above environmental problems, air pollution and water pollution are more destructive to people's daily lives [2]. There is no doubt that the study of the relationship between environmental protection and social development is of great significance [3].

In recent years, the trade-off between environmental protection and social development has become the focus of research in this field. Generally speaking, the research on the relationship between the two is mainly achieved through three aspects: theory of sustainable development (SD), Optimization theory, and new development structure perspective. Relevant research progresses are described as follows [4].

\subsection{Sustainable Development (SD)}

The concept of SD has varied a lot since it was first purposed in 1980. Our common future reflected in a trade-off problem of environment and development equity [5]. Carbon emissions is a hot topic in that the effects of carbon dioxide $\left(\mathrm{CO}_{2}\right)$ are global [6]. Gradually, carbon emissions rights became a kind of strategic resources which thus became a commodity traded between countries [7]. SD is not just slogan, but a set of scientific operation system. Aiming at eliminating waste and the continual use of resources, circular economy was proposed. But it comes with the problem of inadequate infrastructure [8]. Some recent works like [9] start to explicitly model SD in various aspects such as the relationship between urbanization and environment.

\subsection{Optimization theory}

Kuznets curve is an inverted $U$ curve. It was first proposed by Kuznets, an American economist, in 1955. This Curve was named as the Environmental Kuznets Curve (EKC) in the field of environment. Some researchers predicts that emissions of atmospheric pollutants will increase sharply for several centuries based on EKC [10]. Others took Austria as an example, concluded a relatively reliable relation based on EKC [11]. In an engine system, environmental factors are directly included in the optimization of engine efficiency, and two solutions are provided: one is the optimization method based on penalty function, the other is the application of the concept of Pareto Optimality, which is simply a compromise of multiple optimal solutions [12], many economic concepts can be almost perfectly applied to SD problems.

\subsection{New development structure perspective}

Innovations give rise to new development structures. However, new development structure brings about challenges of environment and society [13].

\footnotetext{
${ }^{*}$ Corresponding author: grwen@mail.xjtu.edu.cn
} 
Environment challenge includes the pollution of air and water [14]; the management of rubbish, the impact of climate change [15]. Waste disposal methods are also crucial to the cyclic development, as it is points out by some literature that particular kinds of waste such as plastic can have huge negative effect and will become a dominant problem[16]. The other prominent example comes from the air pollution of China, which is reported by [17] that the on social media, people tend to express less happiness because of this problem. And society challenge includes poverty [18], corruption and the problem of human rights [19]. Energy supply is a safeguard of development, and the key points are energy efficiency and renewable energy [20].

Although the works mentioned above have achieve good analytical results, they either did not quantify the relationship between environment and society or discussed nothing about social development factor at all. Therefore, a framework for analyzing environmental protection and social development was proposed with an optimization perspective in this paper. First, data whitening ands filtering techniques are used to preprocess the data. Second, the correlation between selected features of pre-processed data is analyzed by using Karhunen-Loeve Transformation (K-LT). Then regression is used to quantify the relations between fused factors and optimization objective. Ultimately, we are able to obtain the quotas and strategies for development and environment protection by solving the optimization problem and result from analysis. The contribution in our work can be summarized as follows.

1) An optimized perspective to discuss the relationship between environmental protection and social development was proposed firstly, and the results of interpretability were also verified by experiments.

2) Some features that characterize environmental protection and social development are firstly listed in the proposed model, which are integrated into a unified objective function.

3 ) The solution of the model is able to yield feasible quota for developmental policies and specific criteria for environmental regulations.

4) The data from 2000 to 2018 of China's economic and social development has been adopted to prove the effectiveness of the proposed framework.

The rest of the paper is organized as follows. Section 2 briefly reviews the theoretical foundation of the proposed method, and section 3 introduces the proposed framework in detail. In section 4, the proposed method is used to verify and analyze the realistic case of China from 2000 to 2018. Finally, in section 5 , the conclusion of this paper and the future work are presented.

\section{Theoretical Foundation}

\subsection{Adaptive Robust Kalman Filter (ARKF)}

Statistics of these features from 2010 to 2018 display a definite trend, though, data fluctuate subtly with a year.
Aiming at feature fusion, we make filtering and smoothing based on major tendency of the data. ARKF provides a robust estimation algorithm in the sense of generalized mean square error and thus mitigates the impact of outliers [21]. Normal Kalman filter is a recursive least-square algorithm, mean squared loss function is used as criterion. $M$ estimator is applied in the least-square problem of ARKF.

$$
\sum_{i=1}^{n} x_{i j}(k) \Psi\left(y_{i}(k)-x_{i}^{T}(k) \widehat{\beta}(k)\right) \approx 0
$$

where, $x_{-} i j(k)$ is the element in the $\mathrm{i}$-th row and $\mathrm{j}$-th column of the matrix $X(\mathrm{k})$, and $\Psi(\bullet)$ is the robust function used in $\mathrm{M}$ estimation. Huber function is used here:

$$
\Psi(x)=\left\{\begin{array}{c}
x^{2}, x \leq \Delta \\
2 \Delta x-\Delta^{2}, x>\Delta
\end{array}\right.
$$

where $\mathrm{X}(\mathrm{k})$ here is given as follows:

$$
\begin{gathered}
E(k)=\left(\begin{array}{cc}
\breve{P}(k) & 0 \\
0 & R(k)
\end{array}\right) \\
S(k) S^{T}(k)=E(k) \\
S^{-1}(k)\left(\begin{array}{c}
I \\
H(k)
\end{array}\right)=X(k)
\end{gathered}
$$

where, $\mathrm{P}(\mathrm{k})$ denotes the priori covariance of the state transition in the current iteration, $\mathrm{R}(\mathrm{k})$ is the covariance of the observation noise. Equation (3) is the matrix decomposition of noise covariance matrix. $\mathrm{H}(\mathrm{k})$ is the observation matrix. For the sake of robust kernel function, disturbance brought by outliers are mitigated, which makes the iterative smoothing process more stable in the presence of abnormal fluctuation. ARKF is adaptive in that the adaptive estimation of covariance of noise, instead of a static value used in normal Kalman filter. This estimation is based on the robust mean value of noise, which can be obtained by Huber function:

$$
\sum_{i=k-N+1}^{k} \Psi\left(\frac{r(i)-\hat{r}(k)}{\widehat{d}(k)}\right)=0
$$

where, $r(k)$ is the robust estimation of mean value, $d(k)$ is the absolute median deviation. After the aforementioned procedure, better smoothing effect can be obtained.

\subsection{Karhunen-Loeve Transformation (K-LT)}

K-LT can be used to abstract a reduced dimensional representation from high-dimensional features [22]. We adopt a simpler nuanced expression. The idea is to change the basis of the high-dimensional feature vector $\mathrm{x}$, and thus intercept some basis vectors with denser information:

$$
\widehat{\boldsymbol{x}}=\sum_{i=1}^{d} c_{i} \boldsymbol{u}_{i}=\boldsymbol{c}^{T} \boldsymbol{U}
$$

A minimization of the error between the original vector and the sample vector transformed into a lower 
dimensional space and is needed, i.e., a constraint minimization problem in the case of constraint $\mathrm{C}$ scale:

$$
\begin{gathered}
\min e=E\left[(\boldsymbol{x}-\widehat{\boldsymbol{x}})(\boldsymbol{x}-\widehat{\boldsymbol{x}})^{T}\right] \\
=\sum_{j=d+1}^{+\infty} \boldsymbol{u}_{i}^{T} E\left[\boldsymbol{x} \boldsymbol{x}^{\boldsymbol{T}}\right] \boldsymbol{u}_{i} \\
\text { s.t. } \boldsymbol{u}_{i}^{T} \boldsymbol{u}_{i}=1
\end{gathered}
$$

Solve the least-square problem defined by Equation (8), and the following can be obtained:

$$
E\left[\boldsymbol{x} \boldsymbol{x}^{\boldsymbol{T}}\right] \boldsymbol{u}_{i}=\lambda_{i} \boldsymbol{u}_{i}
$$

where, $\lambda_{i}$ is the eigenvalue of matrix $\boldsymbol{\Phi}=E\left[\boldsymbol{x} \boldsymbol{x}^{\boldsymbol{T}}\right]$. In particular, K-LT is equivalent to principal component analysis (PCA) when $\boldsymbol{x}$ has been decentralized. Equation (8) defines a problem of minimizing the errors brought by the representation in the remaining dimension, and that is why we choose the corresponding eigenvectors $\boldsymbol{u}_{i}$ of $\mathrm{k}$ biggest eigenvalues for transformation.

\subsection{Sigmoid Regression}

Sigmoid function is a function which is widely used in modeling $[23,24]$. Its saturability corresponds with the maximum of satisfaction index, and its trend is also consistent with the principles of economics: when the marginal utility approaches to zero, the total utility reaches its zenith. The range of satisfaction is set as $(0,4)$, a sigmoid-like satisfaction function can be obtained:

$$
\operatorname{SAT}(t)=\frac{4}{1+\exp (-b(t-c))}
$$

where, $t$ represents time or value that is combined with factors, $\mathrm{b}$ and $\mathrm{c}$ are parameters calculated by data fitting.

\section{Proposed Framework}

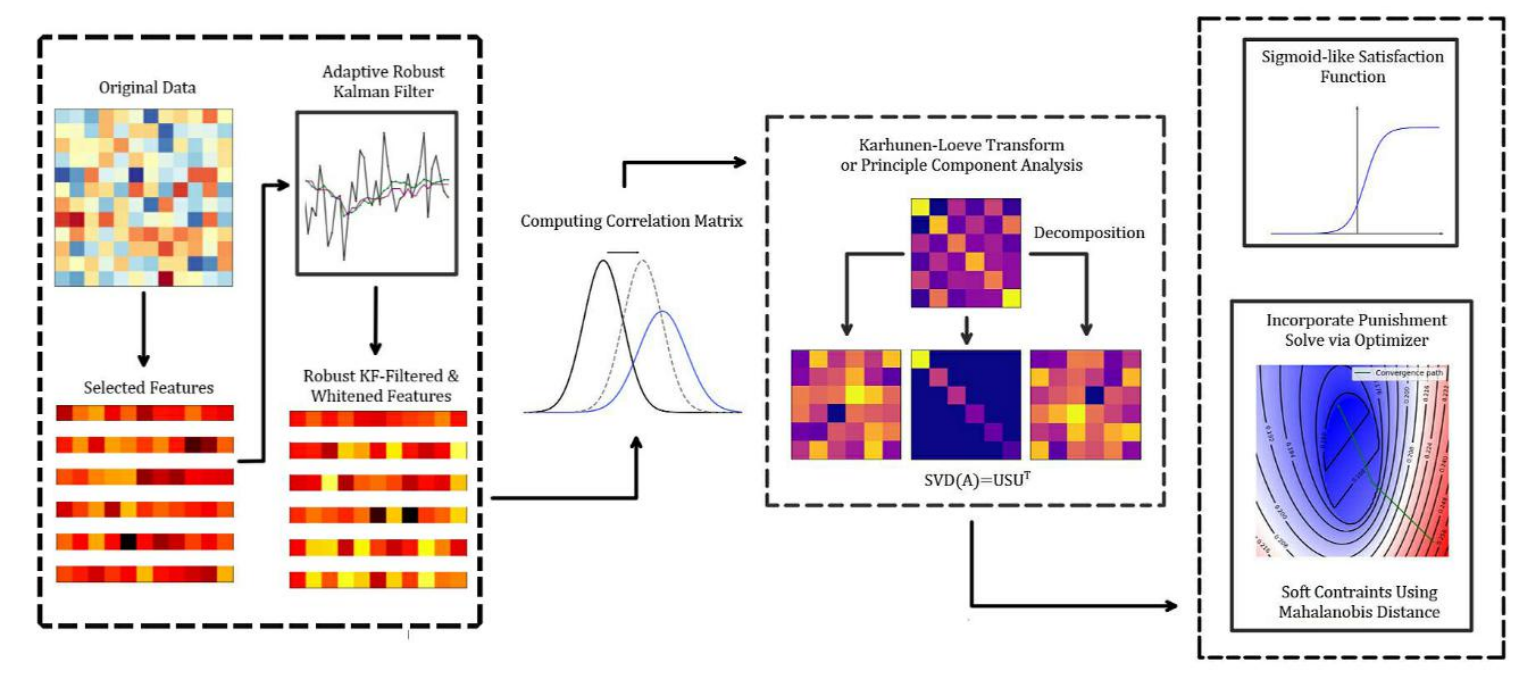

Fig. 1. Proposed analytical procedure framework

(1) Select more representative features from a range of society/ environment indicators.

(2) Make filtering and smoothing at time-domain properties of the features, normalization is needed.

(3) Make correlation calculation or K-LT to get correlation matrix, then make decomposition analysis and thus combine features in low-dimension.

(4) Obtain objective function by regressing the features after combined and Sigmoid average satisfaction function. Penalty term is added, and optimizer is used in the process of iteration computation.

\section{Experiments and Analysis}

\subsection{Objective}

Our optimization objective is:

$$
x_{d}^{*}, x_{e}^{*}, x_{s}^{*}=\underset{x_{d}, x_{e}, x_{s}}{\operatorname{argmin}} f\left(x_{d}, x_{e}, x_{s}, t\right)
$$

where, $x_{d}, x_{e}$ and $x_{s}$ are development, environment and science indicators. All three can be multiple vectors or the scalars after feature combination. $f$ is the objective function, and here it is the satisfaction function. Constraint can be expressed as:

$$
\text { s.t. } \sqrt{(\boldsymbol{x}-\overline{\boldsymbol{x}})^{\mathrm{T}} \Sigma(\mathrm{t})^{-1}(\boldsymbol{x}-\overline{\boldsymbol{x}})} \leq r(t)
$$

where, $r(t)$ is the number of options for humans which decreases with time, and $\Sigma(t)$ is a time-variant correlation matrix. Equation (12) means the Mahalanobis distance must not be bigger than a certain value, and the value of $\Sigma(t)$ in this paper has a noise which is a zero-mean and $\sigma^{2}$-variance random process. 


\subsection{Data Description}

In order to model the three indicators proposed in equation (11), we choose 11 different features of China in 2010-2018 and 2000-2009 to analyze.
Data are listed in the following table. The underlined cells represent the missing data of the year, which is predicted by simple regression. Data from 2000 to 2009 derive from the same sources, and there is no need for data completion, thereby not listed.

Table 1. Feature statistics used in the model (2010 - 2018)

\begin{tabular}{|c|c|c|c|c|c|c|c|c|c|c|c|}
\hline Feature $\backslash$ Time & 2010 & 2011 & 2012 & 2013 & 2014 & 2015 & 2016 & 2017 & 2018 & class & source \\
\hline Life Satisfaction & 3.769 & 3.901 & 3.824 & 3.768 & 3.82 & 3.872 & 3.847 & 3.854 & 3.861 & l & {$[25]$} \\
\hline GDP/Billion \$ & 6087 & 7551 & 8532 & 9570 & 10475 & 11061 & 11233 & 12310 & 13894 & $x_{d}$ & {$[26]$} \\
\hline Income/\$ & $\begin{array}{l}1910 \\
9\end{array}$ & 21810 & 24565 & 26467 & 28844 & 31195 & 33616 & 36396 & 39251 & $x_{d}$ & {$[27]$} \\
\hline Energy Consumption/TWh & 4150 & 4703 & 4966 & 5342 & 5523 & 5550 & 5920 & 6308 & 6840 & $x_{d}$ & {$[28]$} \\
\hline $\mathrm{CO}_{2}$ Emission/(Mt/year) * & 951.8 & 882.2 & 392.7 & 490.1 & 55.91 & 58.76 & 39.92 & 270.4 & 332.4 & $x_{e}$ & {$[29]$} \\
\hline Forest Loss/kha & 5128 & 5029 & 7911 & 5569 & 5131 & 3645 & 3709 & 2380 & 1459 & $x_{e}$ & {$[30]$} \\
\hline PM2.5 exposure $/\left(\mathrm{mg} / \mathrm{m}^{3}\right)$ & 69.48 & 70.54 & 63.83 & 65.51 & 59.77 & 59.06 & 52.21 & 52.66 & 47.90 & $x_{e}$ & {$[30]$} \\
\hline NOx Emission/(Mt/year) & 25.09 & 26.30 & 26.51 & 27.12 & 26.84 & 26.37 & 25.85 & 25.38 & 24.78 & $x_{e}$ & {$[31]$} \\
\hline SO2 Emission/(Mt/year) & 32.07 & 33.05 & 32.46 & 32.34 & 31.88 & 30.90 & 30.44 & 29.53 & 28.04 & $x_{e}$ & {$[31]$} \\
\hline $\begin{array}{l}\text { Renewable energy output } \\
\text { ratio }\end{array}$ & 18.62 & 16.76 & 19.97 & 20.30 & 22.61 & 23.93 & 24.63 & 25.33 & 26.04 & $x_{s}$ & {$[30]$} \\
\hline R \& D expenditure (\% GDP) & 1.714 & 1.780 & 1.912 & 1.998 & 2.030 & 2.066 & 2.118 & 2.145 & 2.186 & $x_{s}$ & {$[30]$} \\
\hline
\end{tabular}

The following analysis is based on the data of the features listed in the table above. The in-class combinations of the three categories correspond to three types of independent variables $x_{e}, x_{d}, x_{s}$.

\subsection{Dimensionality Reduction}

The feature matrix is high dimensional, therefore dimensionality reduction needs to be applied after ARKF filtering, making it easier for regression and visualization. We apply decentralization and normal-ization according to features which compose the factor. K-L transformation can be used due to high within-class correlation, after which we acquire three factors as variables. The visualized results are shown in Fig. 2. Regression is based on the three factors, according to Equation (10). The temporal development of factors can be used to derive satisfaction function using these factors as variables, because time $t$ serves as independent variable in satisfaction function. Further linear combination retains $96.88 \%$ original information, and the combination is written as follows:

$$
t=-0.659 x_{e}+0.585 x_{d}+0.472 x_{s}
$$

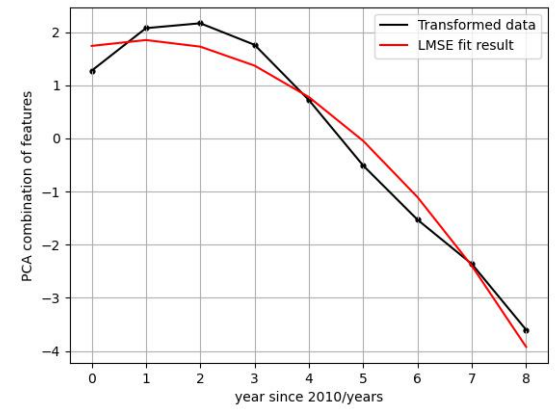

Fused environment feature Retained information: $82.74 \%$

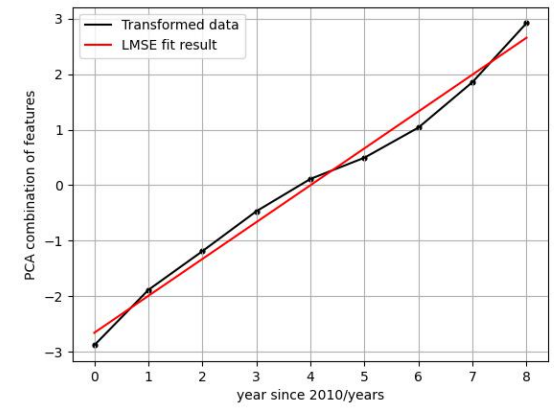

Fused development feature Retained information: $99.29 \%$

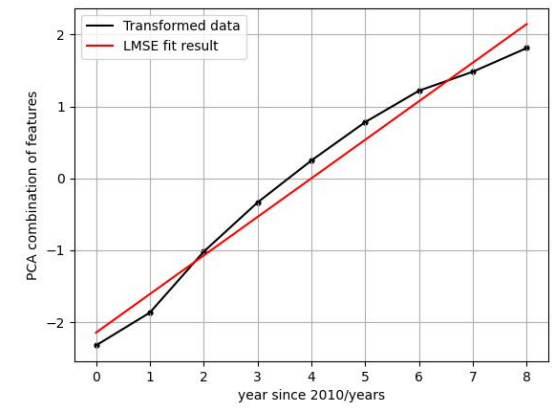

Fused R\&D feature Retained information: $98.21 \%$

Fig. 2. K-L transformation result visualization (2010-2018)

\subsection{Covariate constraint problem}

Environment policies were not strict from 2000 to 2009, which caused worse environmental problem. We can ignore other less significant factors which interfere society and environment interaction during this period. In K-L transformation, signal correlation matrix defined as follows can substitute covariance matrix. 


$$
R_{i, j}=\sum_{i=0}^{T} \boldsymbol{x}_{\boldsymbol{i}}(\tau) \boldsymbol{x}_{\boldsymbol{j}}(\tau)+\lambda \sum_{i=0}^{T} \boldsymbol{x}_{\boldsymbol{i}}(\tau) \boldsymbol{x}_{\boldsymbol{j}}(\tau-1)
$$

where $R_{i, j}$ is the matrix element at row $\mathrm{i}$, column $\mathrm{j} . \lambda$ is assigned manually, implicating the influence of historical values. Equation (14) correlates the two features in the same year and within a one-year span due to Markov property. We multiply the matrix by its transpose counterpart element-wisely for symmetry property after normalizing it to $[-1,1]$ interval. Note that when $\lambda$ is 0 , this matrix degrades to covariance matrix, otherwise it possesses Markov property. Take $x_{d}, x_{e}$ as examples, using K-L transformation based on this correlation matrix, we find that the two variables are covariates. That is to say, environmental requirements constrain development and vice versa. It also vindicates Mahalanobis distance constraint defined in Equation (12).

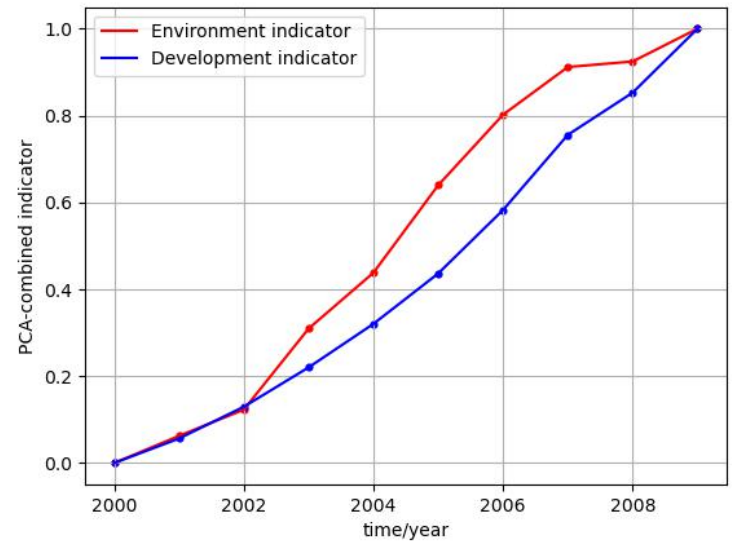

Fig. 3. Correlation K-L dimension reduction (2000-2009)

Considering that the constraint defined in Equation (12) is mathematically complicated, we further redefine the punishment as Equation (15), which punishes the solution outside the constrained range, incorporating it into the objective for gradient calculation. $\eta$ is a assignable parameter and we set it to be 0.01 in this paper.

$$
\begin{aligned}
& d=\sqrt{\left(x-x_{c}\right)^{T} \Sigma^{-1}\left(x-x_{c}\right)} \\
& L(d)=\eta d \text { if } d>r \text { else } 0
\end{aligned}
$$

To make the results more tangible, we fuse $x_{s}$ directly into $x_{e}$ during feature fusion in 4.3 , resulting in two independent variables visualized as follows:

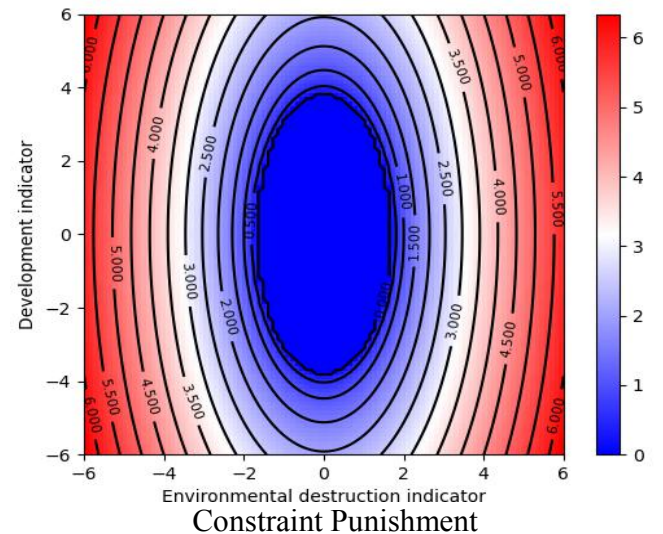

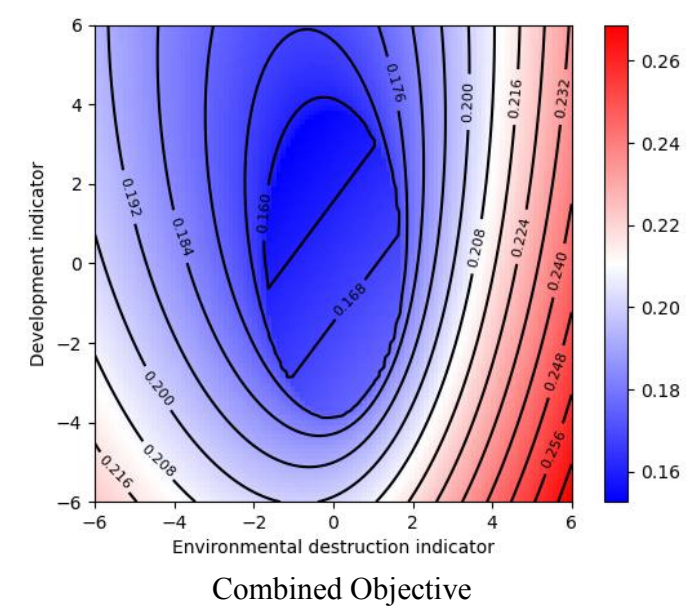

Fig. 4. Objective visualization

Different iterative strategies and updating strategies of learning rate are adopted in the objective function, which corresponds to the exploration of development route in reality as well as the attention and precision of policy modifications. Firstly, we use SGD optimizer, in the way that policy-making considers no historical information or previous experience, while only greedily judges the development direction by relying on the current situation. We set $\Sigma$ matrix in Equation (12) as a two-dimensional diagonal matrix, and the values on the diagonal are: $(1,5)$. The fixed learning rate of the optimizer is 1.0 and 2.0, cor-responding to different development policy modification efforts. It can be found that there will be oscillation when approaching the solution in the first row of Figure 5. Corresponding to the mathematical phenomenon, in reality, it is difficult for the development strategy and related policies to converge on a better plan, therefore constantly carry out tedious fine-tuning. On the other hand, if we dynamically adjust the learning rate according to the situation, the convergence is more stable (shown in the second row of Figure 5), that is, we need to carefully consider when to be radical and conservative.

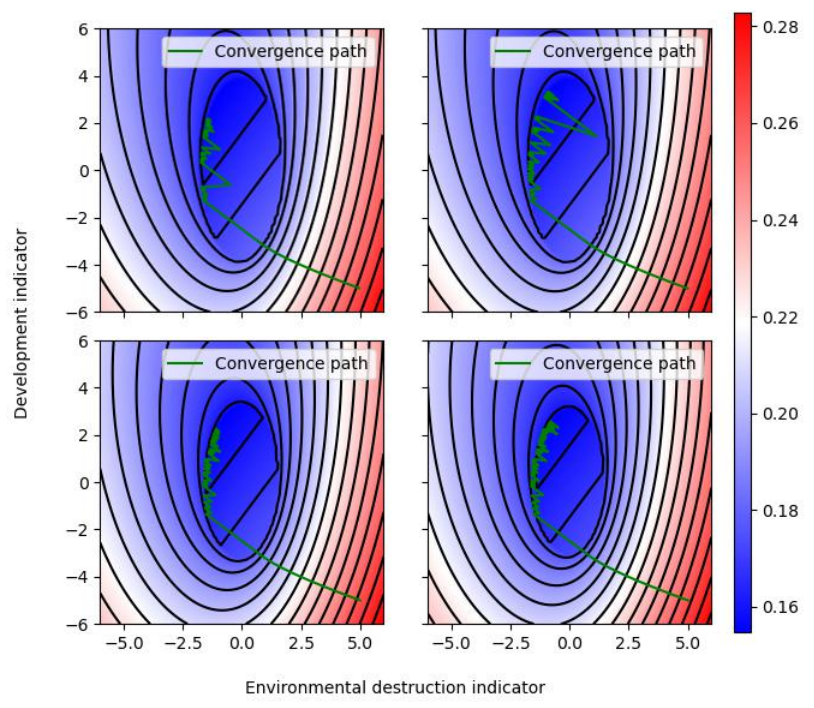

Fig. 5. Dynamic constraint space with fixed learning rate (row 1) and adaptive learning rate (row 2). 


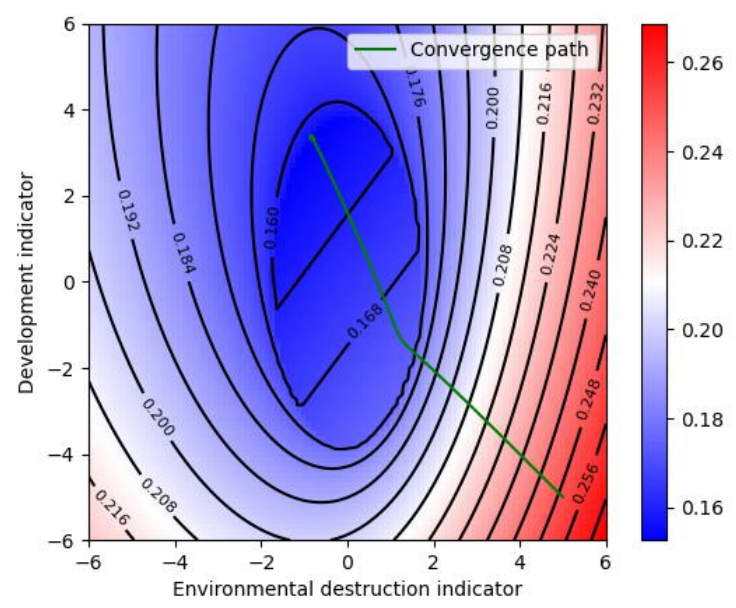

Fig. 6. Adam optimizer with stable optimization process

If we use different optimizers, such as Adam optimizer [32], we can get the result as shown in Figure 6. Compared with SGD, which only depends on current knowledge for optimization, while Adam optimizer has more stable outcome and possesses realistic counterpart as well as mathematical advantages, which can provide us with corresponding development inspirations in this problem:

The learning rate of Adam optimizer is adaptive, and it constantly makes filtering correction according to historical information: the modification of policies and development routes needs to have a certain degree of continuity, therefore we can't only make policies according to the current situation, and it is vital to avoid large-scale modification of policies when the change of people's satisfaction is small.

Adam optimizer has a momentum mechanism, and can naturally carry out annealing steps: a good iterative development strategy is made according to the historical development trend of various indicators, and will not fall into local optimal solution because of conservatism.

\section{Conclusion}

Aiming at the trade-off problem of social development and environmental protection, the issue is modeled as a single objective optimization problem and discussed. Social development, environmental protection and other relatively independent factors are integrated, and based on which we obtain a mathematical expression of the objective-the average satisfaction function of people-which needs to be optimized. Considering the data correlation, we proposed a method for correlation analysis of the features, and dimensionality reduction feature fusion is carried out.

We proposed a model which could be used to obtain the numerical solution of the optimal value of factor under the time-varying constraints brought by environment and policies. On the one hand, this numerical solution corresponds to the specific reference index of the final environment and development policies when the constraints are changing over time. On the other hand, the similarity between the convergence process of solution and the process of actual policy making can be used as a simulation of the development, e.g., the oscillation of solution and the revision of policies.

Besides, the relationship between the mathematical iterative optimization process of the problem and the formulation and revision of policies in reality is discussed, as well as some enlightenment from the mathematical process. Human activity has become more destructive, thus the space of constraints of environment policies is gradually shrinking, and the problem becomes ill-posed which reflected in the deterioration of the optimal value as the solution space deteriorates and the oscillation in the process of solution. The discussion is established from Chinese perspective, but we live in an age of globalization, which prompts a solution to the optimization problem of parallel development before it is badly ill-posed and goes further in the direction of insolvability or meaninglessness. Via the approach of problem parameterization, the fuzzy relations between environment and society can be mathematically modeled, in contrast to the pure literal discussions which provides fewer feasible plans and specific policy criteria.

Making correlation analysis to obtain the expression of dimensionality reduction feature is a crucial step. However, it is based on artificial selection. It will be discussed in more detail in subsequent works about the use of spectral clustering theory and cluster analysis on more society and environment related features, and thus obtain the optimal dimensionality reduction expression based on min-cut of graph, to reduce the limited capability of model generalization brought about by subjective classification and nonideal feature selection.

\section{Acknowledgments}

This work was supported in part by The National Key R\&D Program of China (No. 2020YFB1710002), in part by the National Natural Science Foundation of China (No. 51775409), and in part by the Equipment Pre-research Fund of China (No. 61420030301).

\section{References}

[1] M. Kampa; E. Castanas. Human health effects of air pollution. 151(2), 0-367. (2008)

[2] "Mortality and Morbidity: Mortality in the 20th century". Australian Social Trends (2012)

[3] Antle, John M.; Heidebrink, Gregg. Environment and Development: Theory and International Evidence. Economic Development and Cultural Change, 43(3), 603-625. (1995)

[4] Figge, Frank; Hahn, Tobias. Is green and profitable sustainable? Assessing the trade-off between economic and environmental aspects. IJPE, 140(1), 92-102. (2012)

[5] Langhelle, O. Sustainable development: exploring the ethics of Our Common Future. IPCR20 2, 129-149. (1999) 
[6] B. Friedl, M. Getzner. Determinants of $\mathrm{CO} 2$ emissions in a small open economy. 45(1), 133148. (2003)

[7] Stern N. A blueprint for a safer planet: how to manage climate change and create a new era of progress and prosperity. Random House. (2009)

[8] J. Korhonen, A. Honkasalo, J. Seppälä. Circular Economy: The Concept and its Limitations. (2018)

[9] C. Fang, X. Cui, G. Li, et al. Modeling regional sustainable development scenarios using the Urbanization and Eco-environment Coupler: Case study of Beijing-Tianjin-Hebei urban agglomeration, Science of the Total Environment. 689, 820-830. (2019)

[10] M. Selden, Daqing S. Environmental Quality and Development: Is There a Kuznets Curve for Air Pollution Emissions? 27(2), 0-162. (1994)

[11] B. Friedl, M. Getzner. Determinants of $\mathrm{CO} 2$ emissions in a small open economy. 45(1), 133148. (2003)

[12] A. Lazaretto, A. Toffolo. Energy, Optimization of multi-criterion systems in thermal systems. 29(8), 1139 - 1157. (2004)

[13] Hilgartner, S, Bosk, C.L. The rise and fall of social problems: a public Arenas model. Am. J. Sociol. 94 (1), 53e78. (1988)

[14] M. Greenstone. Environmental regulations, air and water pollution, and infant mortality in India. $\mathrm{Am}$. Econ. Rev. 104 (10), 3038e3072. (2014)

[15] Huang, J., Yu, H., Guan, X., Wang, G., Guo, R.. Accelerated dryland expansion under climate change. Nat. Clim. Change 6 (2), 166. (2016)

[16] Haider T P, Völker C, Kramm J, et al. Plastics of the future? The impact of biodegradable polymers on the environment and on society. Angewandte Chemie International Edition. 58(1), 50-62. (2019)

[17] S. Zheng, J. Wang, C. Sun, et al. Air pollution lowers Chinese urbanites' expressed happiness on social media. Nature Human Behaviour, 3(3), 237-243. (2019)

[18] E. Britt Patterson. poverty, income inequality, and community crime rates., 29(4), 755-776. (1991)

[19] E. Giuliani Human rights and corporate social responsibility in developing countries' industrial clusters. J. Bus. Ethics 133 (1), 39e54. ( 2016)

[20] Gielen, Dolf; Boshell, Francisco; Saygin, Deger; Bazilian, Morgan D.; Wagner, Nicholas; Gorini, Ricardo. The role of renewable energy in the global energy transformation. Energy Strategy Reviews, 24, 38-50. (2019)

[21] Durovic Z. M., Kovacevic B. D. Robust estimation with unknown noise statistics [J]. IEEE Transactions on Automatic Control, 44(6): 1292-1296. (1999)

[22] Everson R., Sirovich L. Karhunen-Loeve procedure for gappy data [J]. JOSA A, 12(8): 1657-1664. (1995)
[23] Y. Xinyou, J. Goudriaan, E. Lantinga, J. Vos, H. Spiertz A flexible sigmoid function of determinate growth [J]. Annals of botany, 91(3): 361-371. (2003)

[24] J. Han, C. Moraga. The influence of the sigmoid function parameters on the speed of backpropagation learning [C] // IWANN. Springer, Berlin, Heidelberg, 195-201. (1995)

[25] P. Wang, L. Cheng-cheng, Impact of Public Financial Expenditure on People' s Happiness: Based on CGSS Data [J] Journal of Shihezi University (Philosophy and Social Sciences), 33(6), 46-58. (2019)

[26] https://www.macrotrends.net/countries/CHN/chin a/gdp-gross-domestic-product

[27] https://www.statista.com/statistics/259451/annualper-capita-disposable-income-of-rural-and-urbanhouseholds-in-china

[28] https://www.statista.com/statistics/302203/chinaelectricity-consumption/

[29] https://climateactiontracker.org/countries/china/

[30] https://data.worldbank.org/

[31] https://ec.europa.eu/info/energy-climate-changeenvironment

[32] D.P. Kingma and J. Ba. Adam: A method for stochastic optimization. arXiv: 1412.6980 (2015) 